\title{
Emergence of small scales in vortex ring collisions
}

\author{
Ryan McKeown, ${ }^{1}$ Rodolfo Ostilla-Mónico, ${ }^{1,2}$ Alain Pumir, ${ }^{3}$ \\ Michael P. Brenner, ${ }^{1}$ and Shmuel M. Rubinstein ${ }^{1, *}$ \\ ${ }^{1}$ School of Engineering and Applied Sciences, Harvard University, Cambridge, Massachusetts 02138, USA \\ ${ }^{2}$ Department of Mechanical Engineering, University of Houston, Houston, Texas 77204, USA \\ ${ }^{3}$ Université de Lyon, ENS de Lyon, Université Claude Bernard, CNRS, Laboratoire de Physique, \\ F-69342 Lyon, France
}

(Received 2 July 2018; published 17 October 2018)

\begin{abstract}
This paper is associated with a video winner of a 2017 APS/DFD Gallery of Fluid Motion Award. The original video is available from the Gallery of Fluid Motion, https://doi.org/10.1103/APS.DFD.2017.GFM.V0037
\end{abstract}

DOI: 10.1103/PhysRevFluids.3.100509

When two vortex rings violently collide head-on, they rapidly become unstable as they develop complex three-dimensional dynamics that result in the vortex cores breaking down into a fine-scale turbulent cloud [1]. Using high-speed flow visualization techniques with a scanning laser sheet, we are able to reconstruct the breakdown of the colliding cores and characterize the iterative cascade that drives emergence of small-scale flow structures, consistent with recent theoretical predictions [2].

As the vortex rings first begin to collide, they flatten along the collision plane and grow radially, restricting the dynamics of the flow to a narrow volume, as shown in Fig. 1. By injecting the vortex cores with fluorescent dye, we visualize how they deform and ultimately break down during the collision. While the radial stretching of the vortex rings is initially uniform, the close-range interactions between the counter-rotating vortex cores lead to the formation and growth
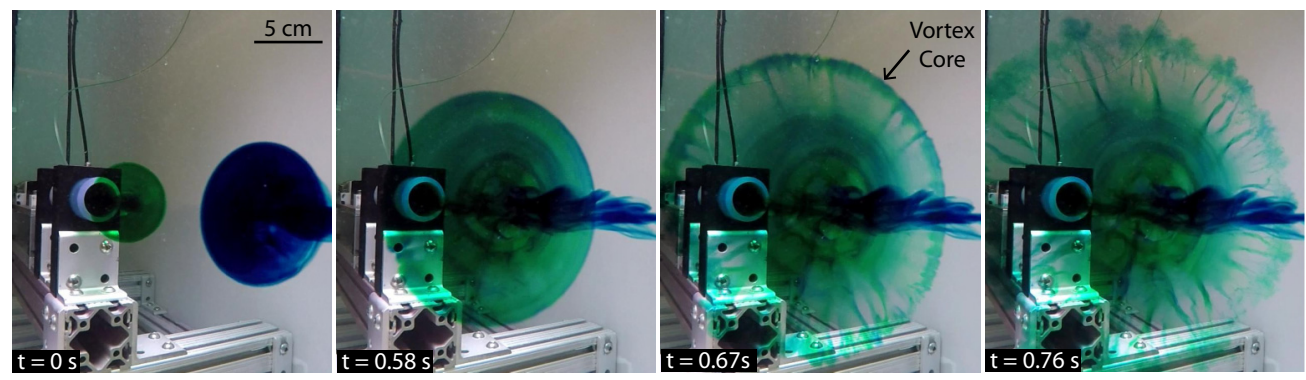

FIG. 1. Head-on collision of two vortex rings. When the vortex rings (dyed with food coloring) collide, they flatten along the collision plane and stretch radially. When the cores contact one another, they rapidly break down into a turbulent cloud.

\footnotetext{
*Corresponding author: shmuel@ seas.harvard.edu
}

Published by the American Physical Society under the terms of the Creative Commons Attribution 4.0 International license. Further distribution of this work must maintain attribution to the author(s) and the published article's title, journal citation, and DOI. 


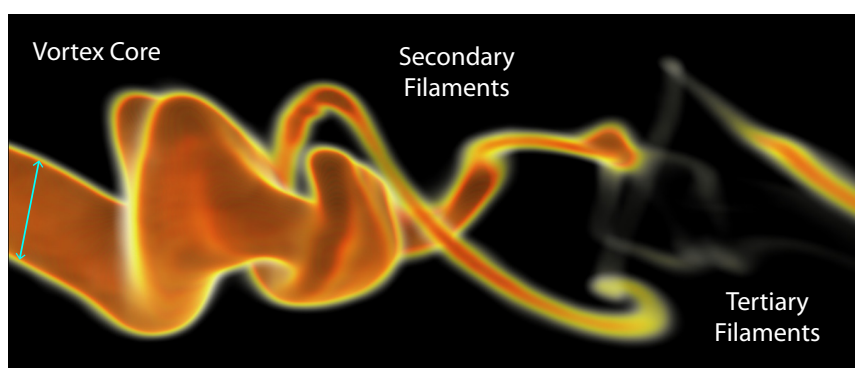

FIG. 2. A cascade of vortex filaments. 3D reconstruction showing how the dyed core breaks down into smaller secondary filaments, which themselves break down into even smaller tertiary filaments. The cyan arrow indicates the size of the core: $2.1 \mathrm{~mm}$.

of long-wavelength perturbations indicative of the Crow instability [3]. The tips of these late-stage perturbations develop a characteristic tentlike structure [2], where the coherent cores contact and rapidly break down. We scan through the flow along the collision plane with a pulsed laser sheet at a rate of 1000 scans per second while imaging the illuminated flow with a synchronized highspeed camera. This tomographic imaging technique allows us to fully reconstruct the intricate, three-dimensional dynamics that result from the breakdown of the colliding cores.

We observe that the breakdown of the vortex cores is initiated by an iterative cascade of instabilities. In order to isolate the flow structures that emerge from the collision, we dye only one of the vortex cores, making the other, undyed core invisible. When the cores are deflected into one another through the development of tentlike structures, the intense strain exerted by the vortices causes the dyed core to locally flatten into an extremely thin vortex sheet. The sheet is stretched by the undyed vortex until it splits into smaller secondary filaments, which begin to unravel the rest of the vortex core. The secondary vortex filaments undergo complicated 3D dynamics as they interact with each other and the other undyed vortex. Upon colliding with other vortices, the secondary filaments, themselves, also flatten break down into even thinner tertiary vortex filaments. The progression of this breakdown is captured beautifully by the snapshot shown in Fig. 2, which illustrates the emergence of secondary and tertiary filaments from the primary vortex core.

The close-range interaction between the cores of the colliding vortex rings leads to the complete breakdown of the vortices, resulting in - via an iterative cascade - a turbulent cloud of many smallscale vortices. The mechanism documented here could therefore be an important ingredient in the turbulent energy cascade whereby the energy of a flow is transferred from large to small scales.

This research was funded by the National Science Foundation through the Harvard Materials Research Science and Engineering Center (Grant No. DMR-1420570) and through the Division of Mathematical Sciences (Grant No. DMS-1715477). M.P.B. is an investigator of the Simons Foundation. S.M.R. acknowledges support from the Alfred P. Sloan Research Foundation.

[1] T. T. Lim and T. B. Nickels, Instability and reconnection in the head-on collision of two vortex rings, Nature (London) 357, 225 (1992).

[2] M. P. Brenner, S. Hormoz, and A. Pumir, Potential singularity mechanism for the Euler equations, Phys. Rev. Fluids 1, 084503 (2016).

[3] S. C. Crow, Stability theory for a pair of trailing vortices, AIAA J. 8, 2172 (1970). 